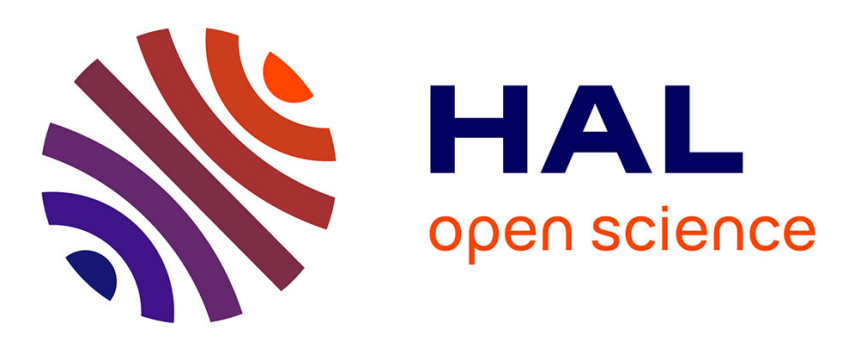

\title{
Motion compensation in two-photon microscopy temporal series
}

Caroline Medioni, Florence Besse, Xavier Descombes, Grégoire Malandain

\section{To cite this version:}

Caroline Medioni, Florence Besse, Xavier Descombes, Grégoire Malandain. Motion compensation in two-photon microscopy temporal series. International Symposium on Biomedical Imaging (ISBI), Apr 2015, Brooklyn, United States. pp.4, 10.1109/ISBI.2015.7164182 . hal-01311755

\section{HAL Id: hal-01311755 \\ https://inria.hal.science/hal-01311755}

Submitted on 4 May 2016

HAL is a multi-disciplinary open access archive for the deposit and dissemination of scientific research documents, whether they are published or not. The documents may come from teaching and research institutions in France or abroad, or from public or private research centers.
L'archive ouverte pluridisciplinaire HAL, est destinée au dépôt et à la diffusion de documents scientifiques de niveau recherche, publiés ou non, émanant des établissements d'enseignement et de recherche français ou étrangers, des laboratoires publics ou privés. 


\title{
MOTION COMPENSATION IN TWO-PHOTON MICROSCOPY TEMPORAL SERIES
}

\author{
Caroline Medioni $^{\star, 1,2} \quad$ Florence Besse $e^{\star, 1,2} \quad$ Xavier Descombes $^{\dagger, 2} \quad$ Grégoire Malandain ${ }^{\dagger, 2}$ \\ * Univ. Nice Sophia Antipolis, CNRS, INSERM, iBV, UMR 7277, 06100 Nice, France \\ ${ }^{\dagger}$ INRIA, 06900 Sophia Antipolis, France
}

\begin{abstract}
Over the past decade, novel live-imaging techniques have considerably changed our vision of cell biology, in particular in the field of neuroscience. Acquisitions of 3D image sequences over long periods of time, in particular, have enabled neurobiologists to follow complex processes such as the development of neuronal populations or degenerative events occurring in pathological contexts, improving our understanding of the mechanisms involved in brain development and function. In most cases, live samples are moving/growing during long-term imaging, therefore it is required to compensate for this global 3D motion before measuring the dynamics of the structure of interest. We present here a method to compute a coherent $3 \mathrm{D}$ motion over a whole temporal sequence of $3 \mathrm{D}$ volumes, which is able to capture subtle sub-voxelic displacements.
\end{abstract}

Index Terms - image registration, two-photon microscopy, neuronal morphogenesis, axonal remodeling

\section{INTRODUCTION}

Quantifying the spatial dynamics of neuronal morphogenesis is crucial to identify the cellular mechanisms and the genetic pathways involved in brain development and repair, and is thus of high priority regarding the increasing number of patients suffering from neurodegenerative diseases. Our work is motivated by the study of developmental axonal remodeling, a genetically-controlled process characterized by a degeneration step followed by a rapid regrowth of axons. Here, we focus our interest on the axonal regrowth phase, which can be studied during brain development, using the fruit fly, Drosophila melanogaster, as a model system [1]. To image axonal regrowth in real time during brain development, we have developed a brain explant strategy and used 2-photon confocal microscopy. Long term imaging reveals a global motion of the sample. To quantify the axonal regrowth event, we have to remove this global motion.

Given a temporal series of images, compensating for a global motion comes to register each image of the sequence to a reference image that can be one image of the sequence. A straightforward approach to compute the motion consists in either co-registering each image of the series with the reference (direct method), or coregistering every pair of successive images and compounding the obtained elementary transformations to build transformations against the reference (indirect method). In both cases, it is assumed that the registration method at hand is accurate enough to capture the sought motion. Temporally distant images may exhibit differences that will impair the registration for the direct method. Moreover, in case of

\footnotetext{
Correspondance: caroline.medioni@unice.fr,gregoire.malandain@inria.fr

${ }^{1} \mathrm{CM}$ and $\mathrm{FB}$ are with "Axon morphogenesis and local RNA regulation in Drosophila" team, iBV, UMR 7277.

${ }^{2} \mathrm{CM}, \mathrm{XD}, \mathrm{FB}$, and GM are with Morpheme project-team, Inria Sophia Antipolis - Méditerranée, I3S, UMR 7271, iBV, UMR 7277.
}

large displacements, the common part between the two images to be registered can be reduced, which may impair the registration too. Thus, the indirect method is often preferred since it comes to coregister images that exhibits few differences, but then compounding transformations is prone to error accumulation.

In our context, it appeared that the registration method at hand is unable to detect the out-of-plane motion between two successive images, since this motion is much smaller than the optical slice thickness. To address this last issue, multiple transformations strategies have been proposed in several contexts: e.g. mosaicking with endomicroscopy [2] or super-resolution microscopy [3]. Such a strategy consists in registering an image of the temporal series against several images of the series (and not only one as in the direct and indirect methods), and in exploiting the redundancy for a better estimation of the transformation against the reference.

We propose here to follow the same kind of scheme to compensate for the the global motion in temporal series in 3D 2-photon confocal microscopy images. In addition, we also propose a quantitative assessment of the compensated series to identify optimal parameters.

\section{DATA DESCRIPTION}

We are following a symmetric population of fluorescently-labeled neurons localized in the central region of the maturing fly brain. This population undergoes a stereotypic developmental degeneration of axons followed by a phase of axonal regeneration, and is thus used as a model to study axonal remodeling. Axonal regrowth generates a distinct projection pattern, allowing the formation of new connections. This event occurs in a time frame of about 15-20 hours, and can thus be followed in real time using two-photon microscopy. The two hemispheres of the brains are imaged here and the regenerating axons are followed thanks to the expression of GFP. A typical acquisition resulted in 3D stacks of 21 planes, each of $1024 \times 512$ pixels, with a pixel size of $0.159667 \mu \mathrm{m}$ and a plane spacing of $0.8 \mu \mathrm{m}$. The imaging field of view is an highly anisotropic parallelepiped of $163.50 \times 81.75 \times 16.8 \mu \mathrm{m}^{3}$. Imaging is repeated every 5 minutes, resulting in time series of about 200 time points. Eleven sequences were used to assessed the proposed method, although only one was used to illustrate the method here.

\section{GLOBAL MOTION COMPENSATION}

\subsection{Registration method}

Registration have been widely addressed in the literature $[4,5]$. It mainly relies on two components: a similarity measure to be optimized and the transformation type to be sought. Similarity measures can be distance-based, when paired features can be identified between the two images to be aligned, or intensity-based (e.g. SSD, 


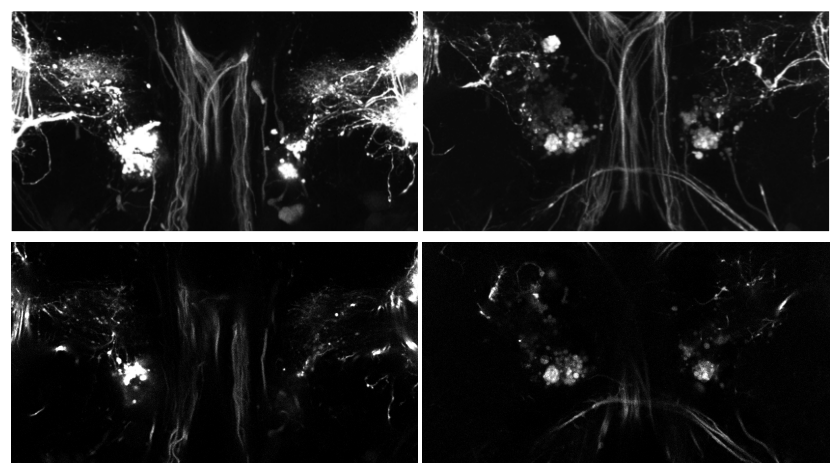

Fig. 1. Top row: left, MIP view of the first 3D stack of a temporal series of 170 time points; right: MIP view of the last 3D stack ; inplane motion is visually obvious while out-of-plane motion can be estimated thanks to the (dis)appearing of some structures. Bottom row: slice \#9 (out of 16) for first and last 3D stacks; it demonstrates the out-of-plane motion.

mutual information). When looking for a global motion, simple transformation types (rigid or affine as in [6]) are generally considered. For a better characterization of spatial dynamics, non-linear transformations may also considered, as in [7]. It can be considered that the linear registration problem is globally solved, and that published linear registration methods are somehow comparable. However, since some changes, due to development, are to be ignored when registering, robust methods have to be preferred [6].

For that reason, we chose to use a block matching scheme [8] already popularized in video coding and versatile enough to address several medical imaging registration problem [9] to compute affine transformations. Such a scheme is comparable to the ICP method [10] except that iconic primitives are matched instead of points.

More precisely, registration aims at the computation of the transformation $T_{f \leftarrow r}$ that will allow to resample a floating image $I_{f}$ onto a reference image $I_{r}$. The transformation $T_{f \leftarrow r}$ is iteratively computed by integrating incremental transformations $\delta T^{t}$, i.e. $T_{f \leftarrow r}^{t+1}=$ $\delta T^{t} \circ T_{f \leftarrow r}^{t}$. At iteration $t$, blocks (or sub-images) $B_{r}$ of the reference image $I_{f}$ are compared to blocks $B_{f}$ of the floating image $I_{f}$, the best block pairing (the one that yields the best iconic measure, here the normalized correlation) yields a point pairing, $\left(C_{r}, C_{f}\right)$, by associating the block centers $C_{r}$ and $C_{f}$. The incremental transformation is then estimated by

$$
\delta T^{t}=\arg \min _{\delta T} \sum\left\|C_{f}-\delta T \circ T_{f \leftarrow r}^{t} C_{r}\right\|^{2}
$$

Linear transformations are computed with a robust method (a Least Trimmed Squares [11] in our implementation but M-estimators are an alternative [12]) that allows to discard outlier pairings.

\subsection{Problem formulation}

Given a time series of $3 \mathrm{D}$ volumes, $I_{1}, \ldots, I_{n}$, compensating for the global motion comes to compute the transformations $T_{i \leftarrow r}$ that allowed to resample the image $I_{i}$ onto a reference image. Here, we sought for affine transformations that can be represented by $4 \times 4$ matrices in homogeneous coordinates:

$$
T=\left(\begin{array}{cccc}
a_{11} & a_{12} & a_{13} & t_{x} \\
a_{21} & a_{22} & a_{23} & t_{y} \\
a_{31} & a_{32} & a_{33} & t_{z} \\
0 & 0 & 0 & 1
\end{array}\right)
$$

Applying the indirect method to our data, i.e. computing transformations $T_{i+1 \leftarrow i}$ or $T_{i \leftarrow i+1}$ and compounding them, allowed to compensated for the in-plane motion, but not for the out-of-plane one. This is exemplified by Fig. 2(a) that depicts the $t_{z}$ component of the affine transformation for the direct method with image \#86 (out of 170 time points) as reference. A plateau appears around the reference corresponding to a null displacement along the $\mathrm{z}$-axis. Indeed the sought $\mathrm{z}$ displacement between any two successive images is below the accuracy of the registration method, and this also stands for all the images in an interval centered on the reference. Picking an other reference just displaces this interval, as illustrated by Fig. 2(b) where images \#43, \#86 and \#128 served as references $\left(t_{z}(86)\right.$ was set to 0 to make curves comparable). When using all images as references and putting together all the $t_{z}$ curves (Fig. 2(c)), it appears that all of them are coherent although different.

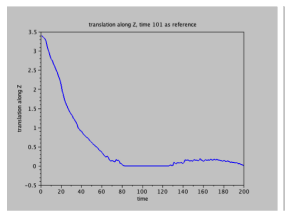

(a)

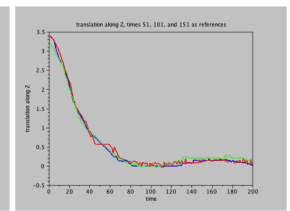

(b)

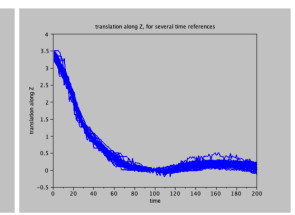

(c)
Fig. 2. The curves depicted the translation parameter along the zaxis for given reference image(s). (a): one reference picked in the middle of the series, a plateau appears in the $\mathrm{z}$ translation parameter around the reference. (b): the previous curve plus the ones for 2 references picked at $1 / 4$ and $3 / 4$ of the series. (c): all images have served as reference.

Let assume we computed a bunch of relative transformations $T_{i \leftarrow j}$ between the images $I_{i}$ and $I_{j}$ of the temporal series, their redundancy can be exploited for the calculation of the sought transformations $T_{i \leftarrow r}$. Indeed, these transformations can be viewed as noisy estimates of composition of the $T_{i \leftarrow r}$ 's, i.e.

$$
T_{i \leftarrow j}=T_{i \leftarrow r} \circ T_{r \leftarrow j}^{-1}+\varepsilon_{i j}
$$

Then, estimating the $T_{i \leftarrow r}$, denoted $T_{i}$ in the following for the sake of simplicity, can be formulated as an least squares problem:

$$
\left\{\hat{T}_{1}, \ldots, \hat{T}_{n}\right\}=\underset{\left\{T_{1}, \ldots, T_{n}\right\}}{\arg \min } \sum_{i, j} d\left(T_{i} \circ T_{j}^{-1}, T_{i \leftarrow j}\right)^{2}
$$

Please note that an adequate norm $d(\cdot, \cdot)$ has to be chosen depending of the considered transformation type.

The resolution of Eq. 4 depends on the transformation type. In [3], authors considered translations for which there exists trivial close form formulations. To our knowledge, there is no such direct solution for linear (rigid or affine) transformations, and Eq. 4 is solved by an iterative optimization. The interested reader will find details for rigid transformation (the difficulty comes from the averaging of rotations) in $[2,13]$. Since we consider that the developing brain may grow globally, we considered here affine transformation and used the $L_{2}$ norm of matrices. To solve Eq. 4, we adapted the iterative resolution scheme of [13].

where $\operatorname{mean}_{d}(\cdot)$ stands for the average computation using the norm $d(\cdot, \cdot)$ (see Eq. 5). After convergence, it is straightforward to express the global motion with respect to any image reference $I_{k}$ by transformation composition: $\hat{T}_{i \leftarrow k}=\hat{T}_{i} \circ \hat{T}_{k}^{-1}$.

$$
\operatorname{mean}_{d}\left(\left\{T_{i \leftarrow j} \circ T_{j}^{(t)}\right\}_{j}\right)=\arg \min _{T} \sum_{j} d\left(T_{i \leftarrow j} \circ T_{j}^{(t)}, T\right)^{2}
$$

\subsection{Implementation details}


Given initial transformations $\left\{T_{1}^{(0)}, \ldots, T_{n}^{(0)}\right\}$;

repeat

$$
\begin{aligned}
& \mid \begin{array}{l}
\text { for } i=1 \ldots n \text { do } \\
\text { end } \\
\quad T_{i}^{(t+1)}=\operatorname{mean}_{d}\left(\left\{T_{i \leftarrow j} \circ T_{j}^{(t)}\right\}_{j}\right)
\end{array} \\
& \text { Regularization of the }\left\{T_{i}^{(t+1)}\right\} \\
& \text { until maximum number of iterations } t_{\max } \text { is reached; }
\end{aligned}
$$

For a practical implementation of the above iterative optimization, several choices have to be made.

1. The initial guess of the transformations $\left\{T_{1}^{(0)}, \ldots, T_{n}^{(0)}\right\}$. Since only small displacements are expected, these transformations are set to the identity transformation. Otherwise, transformations with respect to a reference (direct method) can be chosen too.

2. The choice of the pairwise transformations $T_{i \leftarrow j}$. The more the transformations, the more costly the computations. We pick one image out of 5, i.e. $j \in\{1,6, \ldots\}$ to serve as references and register the neighboring images $I_{i}$ with $i \in[j-N, j+N] \cup[1, n]$ for various values of $N$. When $N$ is equal to the temporal series length $n$, these intervals span the whole series, meaning that all pairwise the $n-1$ registrations against $I_{j}$ are done. We investigated $N \in\{10,30,50,70,100, n\}$.

3. The computation of the average $\operatorname{mean}_{d}\left(\left\{T_{i \leftarrow j} \circ T_{j}^{(t)}\right\}_{j}\right)$. It is nothing but a least squares estimation. Since some registrations may exhibit a large error (particularly if the common support of images to be registered is small), robust estimation (i.e. least trimmed squares) may be preferred.

4. The number of iterations $t_{\max }$. Although the iterative optimization of Eq. 4 is quite fast (because we are dealing with linear transformations; this may not be the case with non-linear ones), performing too many iterations is not critical. However, we have to make sure that enough iterations are done to reach convergence.

5. Regularization of the $\left\{T_{i}^{(t+1)}\right\}$. A continuous smooth global motion is expected. To ensure or enforce the smooth variation of the sought transformations with respect to time, we investigate whether an explicit smoothing step is required or not. We tested a Gaussian regularization with a standard deviation of 10 minutes (the time step between 2 acquisitions is of 5 minutes).

\subsection{Result quantitative assessment}

To compare different parameter settings, it is mandatory to assess/compare quantitatively the results obtained after global motion compensation. If the global motion compensation performs well, the intensity of a point $M$ after compensation should not change during the acquisition duration (axon growth phenomenons are neglected because of their sparsity, and it is assumed that intensity changes due to bleaching are less important that intensity variations due to material displacement), thus that $I_{i} \circ \hat{T}_{i}(M) \simeq I_{j} \circ \hat{T}_{j}(M) \forall i, j, M$ stands, where $I_{i} \circ \hat{T}_{i}$ denotes the image $I_{i}$ resampled by $\hat{T}_{i}$ into the reference frame. The standard deviation of values taken by $M$ (after compensation) over the resampled images $\left\{I_{i} \circ \hat{T}_{i}\right\}$ seemed then to be a relevant objective measure of motion compensation for the point $M$. To assess globally the motion compensation, we computed the probability density function of the standard deviation values. The better the motion compensation, the smaller the standard deviation values. Because of motion, all physical point $M$ imaged in the first image $I_{1}$ may not be imaged during the whole acquisition. We restricted the standard deviation computation to the points belonging to the common acquisition space $\Omega$. Let $S$ be the acquisition space of the 3 -D stacks $I_{i}, S=[1 \ldots X] \times[1 \ldots Y] \times[1 \ldots Z]$, and $\mathbf{1}_{I}$ by the indicator image of the stacks, i.e.

$$
\mathbf{1}_{I}(M)=\left\{\begin{array}{ll}
1 & \text { if } \\
0 & \text { else }
\end{array} \quad M \in S\right.
$$

We have $\Omega=\bigcap_{i} \mathbf{1}_{I} \circ \hat{T}_{i}$. We computed the probability density function (pdf) of the standard deviation of the points $M \in \Omega$ over the whole resampled sequence $\left\{I_{i} \circ \hat{T}_{i}\right\}_{i}$ (see Fig. 3, left). A centile (say 0.8 ) of the probability density function is used as the objective quality measure. The cumulative probability density function, $\operatorname{cpdf}(\cdot)$ (see Fig. 3, right) exemplifies graphically how to assess the motion compensation: the leftmost curve indicates the smaller standard deviation, and $\sigma_{p}$ such that $\operatorname{cpdf}\left(\sigma_{p}\right)=0.8$ is the quality measure for a given parameter settings.
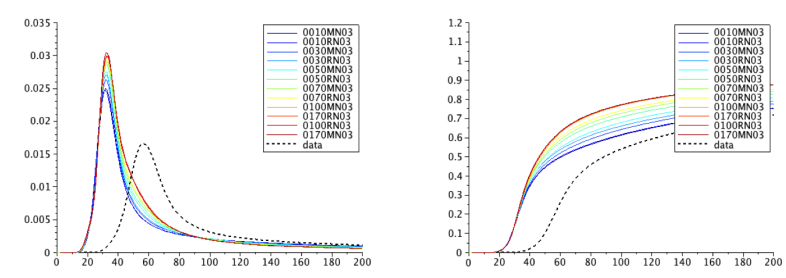

Fig. 3. Left: standard deviation probability density function over the whole resampled sequence $\left\{I_{i} \circ \hat{T}_{i}\right\}$ for several parameter settings (the dashed curve corresponds to original data). Right: cumulative standard deviation probability density function.

\section{RESULTS}

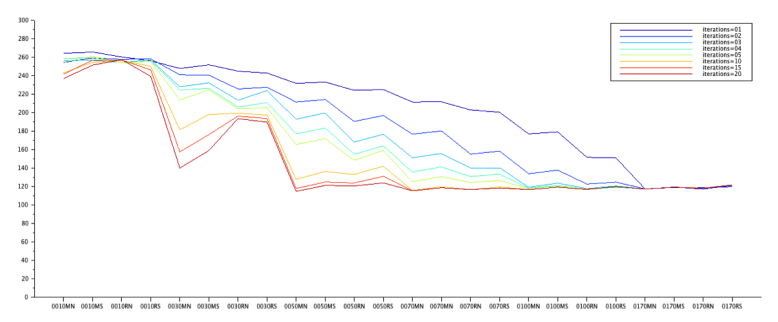

Fig. 4. Quality measures $\sigma_{p}$ for different parameter settings.

Fig. 4 summarizes the quality measures for different parameter settings for one data set. This is also representative for the 20 data sets we have at hand. From our observations, we can derive some general trends:

- There is a floor value for the quality measure: visual inspection of the resampled temporal series reconstructed for this floor value did not reveal any noticeable differences.

- The more the iterations, the faster the convergence towards the floor value.

- The more the transformations $T_{i \leftarrow j}$ (the larger the $N$ ), the faster the convergence towards the floor value.

- Before the floor value is reached, a robust estimation of the transformation average seems to perform slightly better than non-robust estimation, and transformation regularization seems to slightly deteriorate the quality measure. 
From these observations, we choose to perform 20 iterations, to use all pairwise transformations $T_{i \leftarrow j}(N=n)$, without robust estimation of the average nor regularization of the transformation. The evolution of the parameters of the estimated global motion (affine transformations) $\hat{T}_{i}$ with respect to time are depicted in Fig. 5 where they can be compared to pairwise transformations $T_{i \leftarrow j}$. Unsurprisingly, it appears that the parameters involving the third dimension, i.e. the z-axis, are the ones (see e.g. $a_{33}, 3$ rd column and 3rd row) for which there is the more dispersion in the $T_{i \leftarrow j}$.

We choose the first image as reference, and the $\hat{T}_{i}$ 's allow to resample the images $I_{i}$ 's onto $I_{1}$. Fig. 6 presents the resampled last image, $I_{170} \circ \hat{T}_{170}$, which is to be compared to the first one $I_{1}$. It demonstrates visually the global 3D motion compensation.
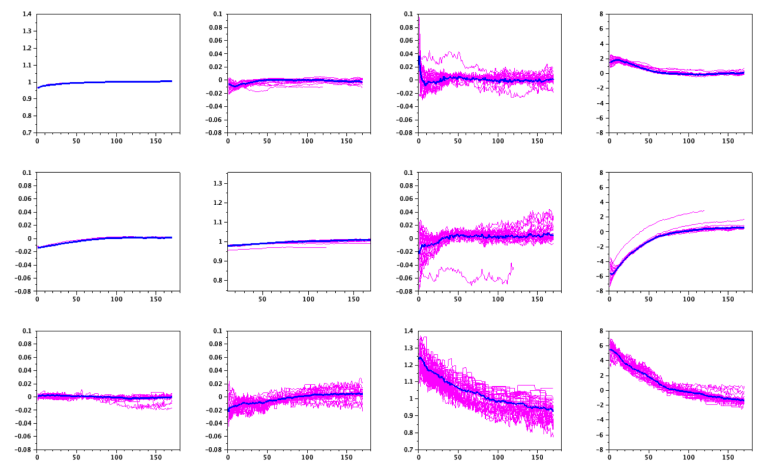

Fig. 5. Purple curves depicted the evolution of pairwise transformation parameters (one curve pear each element of the 3 upper rows of matrix $T$, see Eq. 2; the last row corresponds then to the translation component) for all references, while blue curves depicted the motion estimation.

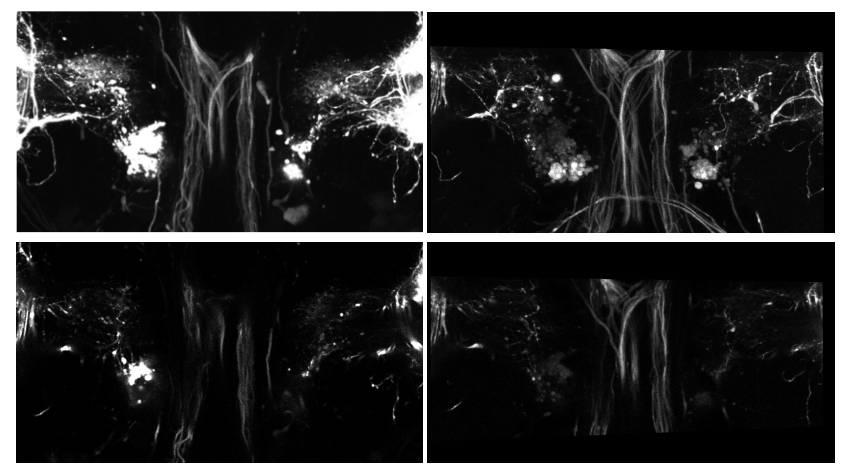

Fig. 6. Top row: left, MIP view of the first 3D stack of a temporal series of 170 time points; right: MIP view of the last resampled 3D stack; in-plane motion correction can be visually estimated. Bottom row: slice \#9 (out of 16) for first and last resampled 3D stacks; the out-of-plane motion has been quite well compensated since similar structures appear in both slices.

\section{CONCLUSION}

We propose a method for global motion compensation that is based on multiple transformations averaging. The proposed results demonstrated its ability to correct for sub-voxelic displacement in temporal series. Moreover, its versatile design makes it able to handle a variety of transformation classes. In addition, we also propose a quality measure for compensated series. This allowed us to objectively compare different parameter settings and identify a default setting for data processing. Thanks to the drift correction, we are now able to quantitatively measure the axonal regrowth without being perturbed by the global motion of the sample. This opens new perspectives to understand how axons are able to regenerate both in normal developmental and in pathological context.

Acknowledgments: this work was supported by the French Government (National Research Agency, ANR) through the "Investments for the Future" LABEX SIGNALIFE: program reference \# ANR11-LABX-0028-01

\section{REFERENCES}

[1] S. Lenz, P. Karsten, J.B. Schulz, and A. Voigt, "Drosophila as a screening tool to study human neurodegenerative diseases," J Neurochem, vol. 127, no. 4, pp. 453-60, November 2013.

[2] T. Vercauteren, A. Perchant, G. Malandain, X. Pennec, and N. Ayache, "Robust mosaicing with correction of motion distortions and tissue deformation for in vivo fibered microscopy," Medical Image Analysis, vol. 10, no. 5, pp. 673-692, 2006.

[3] Y. Wang, J. Schnitzbauer, Z. Hu, X. Li, Y. Cheng, Z.L. Huang, and B. Huang, "Localization events-based sample drift correction for localization microscopy with redundant crosscorrelation algorithm," Opt Express, vol. 22, no. 13, pp. 15982-91, June 2014.

[4] J.B. Maintz and M.A. Viergever, "A survey of medical image registration," Med Image Anal, vol. 2, no. 1, pp. 1-36, 1998.

[5] B. Zitová and J. Flusser, "Image registration methods: a survey," Image Vis Comput, vol. 21, no. 11, pp. 977-1000, 2003.

[6] S. Ozeré, P. Bouthemy, F. Spindler, P. Paul-Gilloteaux, and C. Kervrann, "Robust parametric stabilization of moving cells with intensity correction on light microscopy image sequences," in ISBI, 2013, pp. 464-467.

[7] D. Sorokin, M. Tektonidis, K. Rohr, and P. Matula, "Non-rigid contour-based temporal registration of $2 \mathrm{~d}$ cell nuclei images using the navier equation," in ISBI, 2014, pp. 746-749.

[8] J.R. Jain and A.K. Jain, "Displacement measurement and its application in interframe image coding," IEEE Trans. Commun., vol. 29, pp. 17991808, 1981.

[9] S. Ourselin, A. Roche, S. Prima, and N. Ayache, "Block matching: A general framework to improve robustness of rigid registration of medical images," in MICCAI. 2000, vol. 1935 of LNCS, pp. 557-566, Springer.

[10] P.J. Besl and N.D. McKay, "A method for registration of 3-D shapes," IEEE Transactions on Pattern Analysis and Machine Intelligence, vol. 14, no. 2, pp. 239-256, February 1992.

[11] P.J. Rousseeuw and A.M. Leroy, Robust Regression and Outlier Detection, John Wiley \& Sons, New-York, 1987.

[12] J.M. Odobez and P. Bouthemy, "Robust multiresolution estimation of parametric motion models," J Vis Commun Image Represent, vol. 6, no. 4, pp. 348-365, 1995.

[13] R. Hartley, J. Trumpf, Y. Dai, and H. Li, "Rotation averaging," Int J Comput Vis, vol. 103, no. 3, pp. 267-305, 2013. 